\title{
Optimization of the large titanium sublimation pumps for the neutral beam injection system on AUG and W7-X
}

\author{
G. Orozco, M. Froeschle, B. Heinemann, C. Hopf, R. Nocentini, R. Riedl and the NBI-Team \\ Max Planck Institute for Plasma Physics \\ Boltzmannstrasse 2, 85748 Garching, Germany \\ guillermo.orozco@ipp.mpg.de
}

\begin{abstract}
The neutral beam injection (NBI) system for the magnetic confinement experiments ASDEX Upgrade (AUG) and Wendelstein 7-X (W7-X) require a vacuum system with high pumping speed of up to $3000 \mathrm{~m}^{3} / \mathrm{s}$ for $\mathrm{H}_{2}$ and large capacity with a gas feed of up to $30 \mathrm{~Pa} \mathrm{~m}^{3} / \mathrm{s}$ and $10 \mathrm{~s}$. It must ensure a vacuum level of a maximum of $10^{-2} \mathrm{~Pa}$ in any section of the neutral beam line in order to avoid re-ionization losses. The large titanium sublimation pumps (TSP) of the NBI System at AUG have been proven suitable for operation within the permanent stray magnetic field of $\mathrm{W} 7-\mathrm{X}$ (up to $30 \mathrm{mT}$ ) if they are operated with 200-500 $\mathrm{Hz}$ sine AC instead of DC for the ohmic heating of the $4 \mathrm{~m}$ long hanging pairs of titanium sublimators [1]. During each heating cycle the pair of sublimators experiences a reversible thermal expansion of around $50 \mathrm{~mm}$ and additionally the tantalum core of the sublimators is subject to material creep, leading to residual lengthening. When this lengthening reaches $70 \mathrm{~mm}$ after 80-100 thermal cycles (3.5-4.5 h operation time) the wires contact the bottom of the NBI vessel causing a short circuit. In order to extend the availability and operation time of the TSP a modification of the sublimator with a tantalum-tungsten core with $2.5 \% \mathrm{~W}$ is being tested, for which less creep is expected. The first results confirm a reduction of the creep rate by a factor of $\sim 20$. This allows for sublimation of the whole Ti inventory without intermediate maintenance of the TSP. Furthermore, possible short-circuit mitigation strategies are discussed.
\end{abstract}

Keywords-Wendelstein 7-X; ASDEX-Upgrade; Neutral Beam Injection; Vacuum technology; Titanium sublimation pump

\section{INTRODUCTION}

Neutral beam injection (NBI) is one of the heating systems used in experimental fusion devices based on the principle of magnetic plasma confinement. At IPP Garching an NBI system is operated on the ASDEX Upgrade (AUG) tokamak since 1993 and a replica system is being built at IPP Greifswald for the stellarator type device Wendelstein 7-X (W7-X) [2]. The AUG-NBI system consists of two injectors with four positive ion sources each, injecting together up to $13 \mathrm{MW}$ of $\mathrm{H}^{0}$ or $20 \mathrm{MW}$ of $\mathrm{D}^{0}$ for a maximum of $10 \mathrm{~s}$. A pressure below $10^{-}$ ${ }^{2} \mathrm{~Pa}$ is required along the path of the neutral beam in order to avoid high re-ionization losses [3], while up to $30 \mathrm{~Pa} \mathrm{~m}^{3} / \mathrm{s}$ of gas $\left(\mathrm{H}_{2} / \mathrm{D}_{2}\right)$ is introduced through the ion sources and in order to neutralize the ions during the $10 \mathrm{~s}$ long NBI pulses. Titanium sublimation pumps (TSP) provide fast pumping speeds of up to $3 \times 10^{3} \mathrm{~m}^{3} / \mathrm{s}$ for $\mathrm{H}_{2}$ at $10^{-2} \mathrm{~Pa}$. In AUG, the TSPs are operated with DC in between plasma discharges, when the magnetic field coils are off. W7-X is designed for long pulse operation with superconducting coils that will remain permanently active [4]. In their stray B-field of up to $30 \mathrm{mT}$ DC operation of the TSP is not possible since Lorentz forces would strongly deflect the $4 \mathrm{~m}$ long $\mathrm{Ti}$ sublimation wires leading to short-circuits with the chamber walls. In an attempt to minimize the amplitude of deflection AC operation of the TSP was successfully tested in a perpendicular B-field of $39 \mathrm{mT}$ in a dedicated test stand [1]. The tests concluded with the definition of a standard sublimation cycle for AC operation of the TSP for the NBI on W7-X based on the DC standard heating cycle implementing sine waveform with frequency ramped from 200 to $500 \mathrm{~Hz}$. For both AC (W7-X) and DC (AUG) operations a solution is sought in order to reduce the frequency of the maintenance periods of the TSP and extend its operation time.

\section{II.THE IPP NBI LARGE TITANIUM SUBLIMATION PUMP (TSP) AND ITS AVAILABILITY}

\section{A. TSP description}

The IPP TSPs have been in operation for the last 20 years in the AUG NBI with four TSPs per injector. The metal sublimation technology provides a large capacity for $\mathrm{H}_{2} / \mathrm{D}_{2}$ and residual gases with a high pumping speed for $\mathrm{H}_{2}$ of up to $700 \mathrm{~m}^{3} / \mathrm{s}$ at $10^{-2} \mathrm{~Pa}$ per pump $\left(2.8 \times 10^{3} \mathrm{~m}^{3} / \mathrm{s}\right.$ in total $)$, fulfilling the NBI system requirements [5]. The titanium sublimes from ohmically heated wires (also called sublimators) and condenses on the surrounding surfaces, building fresh metallic layers that getter the gas molecules. For high pumping speed and capacity a large total surface is needed. This is achieved by the large physical dimensions of the pumps of $4.2 \times 1.5 \mathrm{~m}^{2}$ and the division into nine chambers by $0.25 \mathrm{~m}$ wide separating walls. Additionally, the water cooled walls have corrugated surfaces that further increase the effective surface (fig. 1). When the Ti layer is saturated after some time, a fresh Ti layer is deposited to restore the pumping effect of the layer.

The IPP TSP is designed for long term operation by having a large Ti reservoir. Each chamber houses six $4 \mathrm{~m}$ long sublimators hanging from the top flange, operated in pairs and 


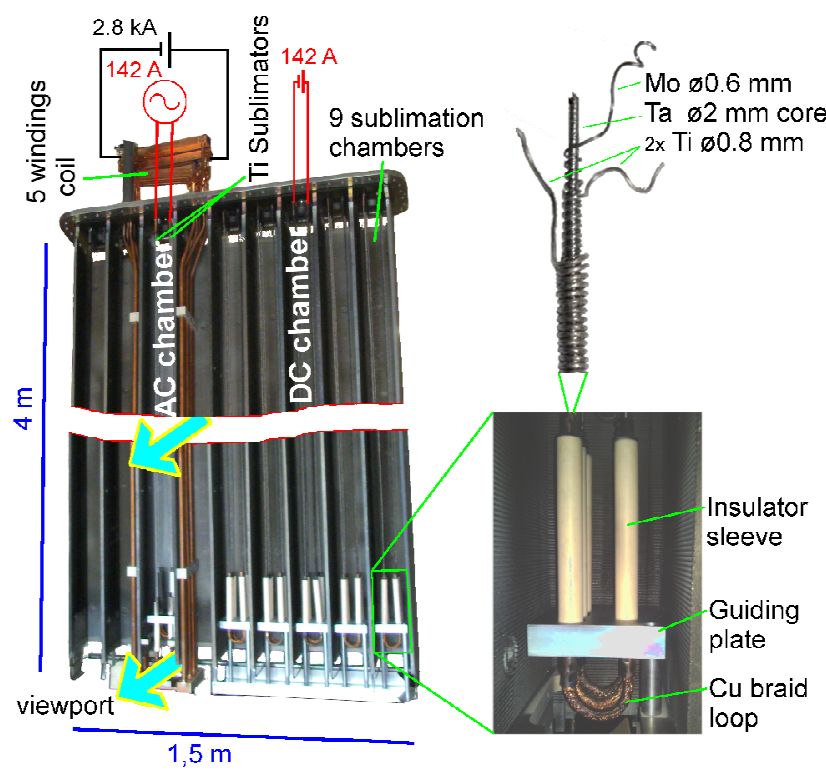

Fig. 1. Set up of the test bed for AC Operation of the TSP. It consists of a modified TSP with a 5 windings planar coil around the chamber for AC operation in B-field

arranged in three rows. The sublimator is made out of two $\varnothing 0.8 \mathrm{~mm}$ titanium wires wound around an $\varnothing 2 \mathrm{~mm}$ tantalum core with an $\varnothing 0.6 \mathrm{~mm}$ molybdenum wire wound in between for stabilization and thermal enhancement (Fig. 1). The design of the sublimator, developed in the 1970s at the French agency CEA [6], contains approximately $200 \mathrm{~g}$ titanium on $4 \mathrm{~m}$ length, which is almost half the weight of the wire. Two sublimators are connected at the bottom with a $\mathrm{Cu}$ braid (Fig. 1). Due to thermal expansion with the temperature rise, the Ta core of the sublimator elongates around $50 \mathrm{~mm}$ during heating cycles. During this expansion the ceramic insulator sleeves at the bottom of the sublimators slide through the holes of a guiding plate. From cycle to cycle the sublimators do not completely return to the original length due to material creep of the Ta core. The residual lengthening accumulates until the $\mathrm{Cu}$ braid loop contacts the floor of the box during a heating cycle, creating a short-circuit. Before this happens, it is switched to the next pairs of sublimators (next row) of the chamber.

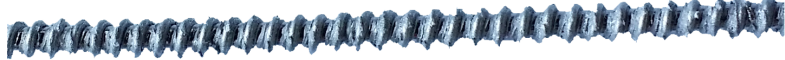
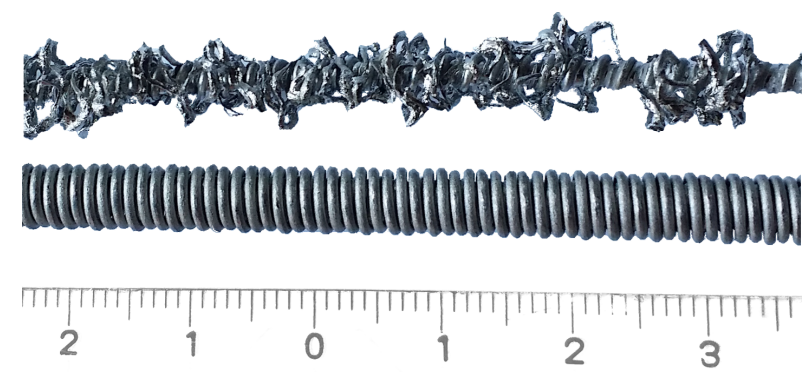

Fig. 2. Ti sublimator use stages. Bottom: sublimator as it installed. Top: sublimator completely depleted of titanium. Middle: advanced stage towards depletion with chaotic but partially connected $\mathrm{Ti}$ wire structures

\section{B. TSP availability}

According to the experience on AUG the Ti content on the wire allows for operation up to $21 \mathrm{~h}$ (Fig. 2). However, the residual lengthening of the wires impedes their operation much before. At IPP in the last 10 years there have been two qualities of sublimators regarding their creep behavior which defines their operation time (Table 1).

TABLE I. OPERATION TIME OF THE TI SUBLIMATORS

\begin{tabular}{|c|c|c|}
\hline \multirow{2}{*}{ Sublimator status } & \multicolumn{2}{|c|}{ Sublimator kind } \\
\cline { 2 - 3 } & Long lived sublimator & Short lived sublimator \\
\hline New & $\sim 7 \mathrm{~h}$ & $\sim 4 \mathrm{~h}$ \\
\hline After $1^{\text {st }}$ shortening & $\sim 15 \mathrm{~h}$ & $\sim 8 \mathrm{~h}$ \\
\hline After $2^{\text {nd }}$ shortening & - & $\sim 8 \mathrm{~h}$ \\
\hline
\end{tabular}

There is unfortunately no explanation for the better performance of the long lasting sublimators used in the past. The sublimators were always manufactured under the same specification by different suppliers and the process was never specifically tracked. Since the Ti reservoir is still large after the pairs of wires contact the bottom they are shortened maintenance phases for further operation. Unfortunately, shortcircuits also occur quite often due to contact between the wires within a pair or with neighboring wires in a chamber. This can render the whole chamber permanently useless if all 3 pairs of sublimators are affected (Fig. 3). The consequence is a loss of useable Ti reservoir of the wires and - even more importantly - a loss of active pumping surface.

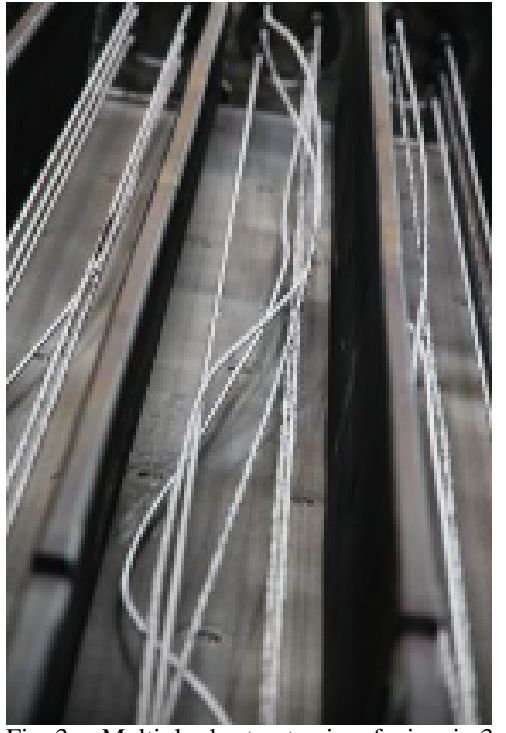
neighboring chambers in a TSP at the end of the last campaign 2014.
Fig. 3. Multiple short-cut pairs of wires in 3

The availability of the pump can be defined as the number of operation hours that can be obtained from all the wires of a TSP. The operation time of a wire depends mainly on the Ti reservoir and its remaining lengthening until contact with the bottom occurs.

\section{TSP availability during the last campaign in AUG}

The results obtained for the last campaign in the NBI-AUG for both injectors (SE: south-east, NW: north-west) are shown in figure 4 . The $\mathrm{x}$-axis gives the operation time accumulated by all sublimators in the pump since installation. The vertical axis indicates the expected remaining operation time of the TSP. At the start point, this value depends solely on the quality of wires installed and their initial condition (new or shortened). Each 


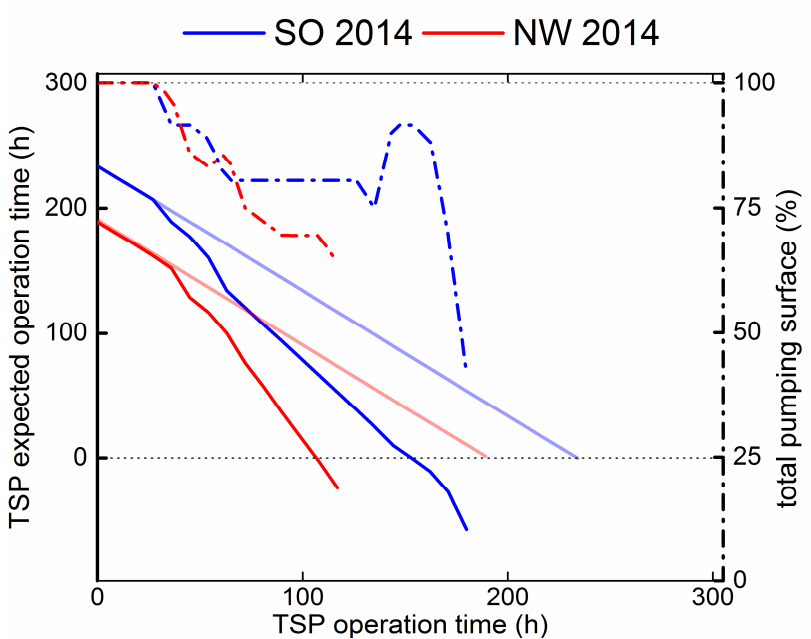

Fig. 4: TSP availability in the two NBI injectors on AUG (SO, NW) for the 2014 campaigns. The solid lines show the real operation which was affected by some sublimator losses. The pale straight solid lines indicate the expected decrease if no short circuits had occurred. The dash-dotted lines indicate the available pumping surface in percent of the total pump surface.

new pair of short-lived sublimators adds four expected operation hours and each shortened wire adds eight (shortlived) or fifteen (long-lived) hours. For the ideal case, when no sudden short-circuits among wires occur, the start operation hours can be completely exploited (straight pale lines). In reality, as the solid lines in Fig. 4 show, the expected remaining operation time decreases faster as pairs of sublimators stick together and fail. If some wire pairs exceed the expected time to contact with the bottom the remaining operation time can also become negative, as is the case for both in injectors in Fig. 4.

Apart from the loss of operation time, the efficiency of the TSP is decreased by the loss of pumping surface (right vertical axis and dashed lines in figure 4). In the last campaign, after 25-30 h of TSP operation the first chambers in both injectors started to fail. Soon after other pairs of sublimators followed, resulting in a reduction of the pumping surface below $80 \%$ rather quickly. By switching to the next row of sublimator pairs the lost pumping surface can be recovered, provided that the contact had not affected neighboring rows.

The TSP availability and its performance for the NBI experiments in AUG have normally been sufficient for the durations of the experimental campaigns, although sometimes unscheduled maintenance was required during campaigns. On a regular operation day of AUG around 20-25 AUG discharges using NBI are performed with $5 \mathrm{~min}$ Ti sublimation in each pump after each discharge and a longer evaporation in every chamber at the beginning of the day. This leads to typically $2.8 \mathrm{~h}$ of TSP operation in every of the four TSPs per day. $\mathrm{W} 7-\mathrm{X}$ is a quasi-steady-state device that will allow for up to 30 min long plasma discharges. The most optimistic NBI operating scenarios consider a full power neutral beam $(10 \mathrm{~s}$, 13-20 MW) every $5 \mathrm{~min}$; or alternatively longer and more frequent pulses at proportionally lower power by switching among ion sources [2]. These operation schemes require a more intensive use of the TSPs. Assuming roughly 70 beam pulses per day one arrives at an estimate of almost $7 \mathrm{~h}$ of TSP operation time in every TSP per day, more than twice as much as on AUG.

\section{EXPERIMENTS FOR THE ENHANCEMENT OF THE TSP AVAILABILITY}

Triggered by the need of the construction of new TSPs for $\mathrm{W} 7-\mathrm{X}$ and given the experimental opportunity provided by the AC/DC TSP test stand a number of improvements are envisioned that might increase the availability and the efficiency of the TSP and reduce the maintenance needs. As wire lengthening and short-circuits between wires have been identified as the main limiting factors for operation time we focus on these issues.

The tests have been performed with AC operation in a B-field at the dedicated test bed facility [1]. It consists of a modified TSP with one of the nine sublimation chambers used for $\mathrm{AC}$ and another for $\mathrm{DC}$ operation. Inside the $\mathrm{AC}$ chamber an elongated five windings coil creates a B-field perpendicular to the wires of strength comparable to the stray B-field at the NBI pumps on W7-X. At the sides of the NBI box, windows have been installed behind the TSP at the bottom and at mid height that allow for direct observation of the sublimators and their lengthening (Fig. 1, viewports).

\section{A. Wire lengthening reduction}

The reduction of the wire lengthening can be achieved by using a substitute material to the supporting core. The boundary conditions for the sublimator are twofold: it must have the same Ti reservoir and the same electrical-power-totemperature relationship in order to use the same power supplies and operational parameters. So far, the $\emptyset 2 \mathrm{~mm}$ Ta core of the sublimator is made of pure Ta. The cyclic thermal loading on pure Ta has been reported to produce a much

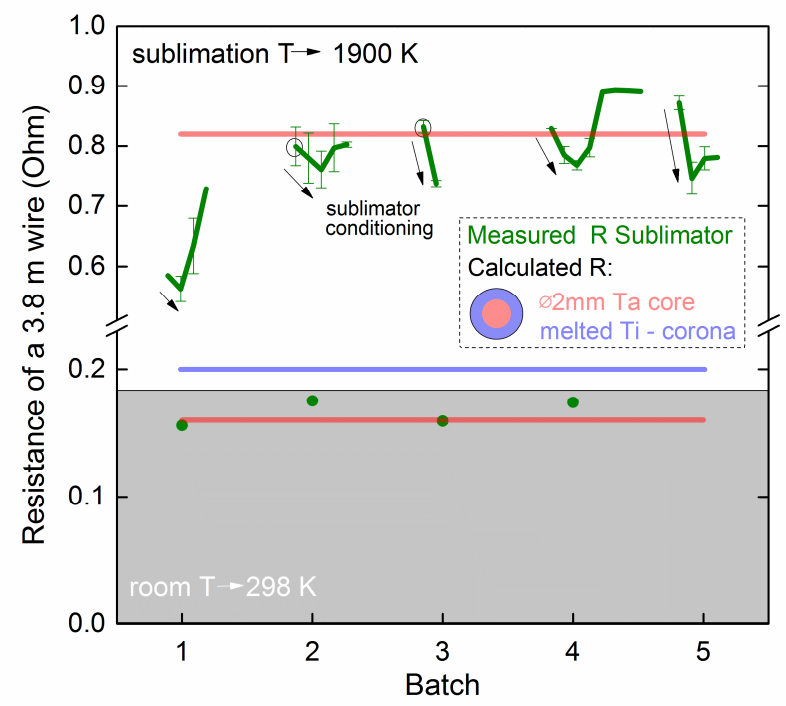

Fig. 5. Electrical resistance of different sublimators in its operation length $(\sim 3.8 \mathrm{~m})$ for 5 different batches (batch number 4 corresponds to the new TaW2,5\% sublimators). They have been measured at room temperature and during operation operated with $\sim 142$ A along its operation live. The measurements evidence that the main conductor is the Ta core. 
stronger creep effect than the one obtained for long maintained thermal loads [7]. However, some TaW alloys have higher resistance against thermal creep of up to a factor of 100 than pure $\mathrm{Ta}$ at maintained heat loads [8]. Depending on the $\mathrm{W}$ content, these TaW alloys have a slightly higher electrical resistivity than pure $\mathrm{Ta}$ at the typical TSP operation temperature of $1900 \mathrm{~K}$ [9] [10]. A TaW alloy with the lowest solute content commercially available of $2.5 \%$ has been chosen to manufacture a number of sublimators for the test.

\section{1) Results for wire electrical properties}

The electrical resistance has been measured at room temperature and then during operation. At the beginning of their operation, the sublimators are conditioned. They are heated to above $\mathrm{Ti}$ sublimation temperature so that the $\mathrm{Ti}$ partially melts and builds a partial corona around the sublimator that improves the $\mathrm{Ti}$ sublimation. As a result, the electrical resistance is reduced slightly between 5 and $10 \%$ of the initial value. However, as is evidenced in Fig. 5 the main conductor is still the Ta core. The spread of the resistance among of the pairs of sublimators of the same batch is also relatively large and depends on effects occurring during the manufacturing process. Furthermore, the resistance changes during the sublimator life due to lengthening and mass change, as more and more $\mathrm{Ti}$ is sublimed. The electrical resistance of the two pairs of sublimators with the new TaW2.5\% core wires (batch number 4 in Fig. 5) lies within the spread of the values obtained for the regular wires used in previous AC operation tests and those used on AUG.

\section{2) Results for wire lengthening}

The elongation of the sublimators is monitored by observing the position of the lowest point of the $\mathrm{Cu}$-braid connection during the sublimation cycle through a window at the bottom of the vacuum vessel behind the TSP (see Fig. 6). After 20-30 s from the cycle start, the sublimators reach thermal equilibrium at nominal current and the $\mathrm{Cu}$ braid loop is then at its lowest point. The distance to a "zero" reference (chosen as the bottom of the back plate of the pump D1 Fig. 6) is measured in each heating cycle.

Figure 6 shows a comparison of the residual lengthening between the two pairs of wires with the new TaW2.5\% core tested so far and the standard wires. For newly used standard sublimators (blue line Fig. 6) the lengthening rate due to creep is $45 \mathrm{~mm} / \mathrm{h}$ during the first operation hour and $\sim 30 \mathrm{~mm} / \mathrm{h}$ during the second. At around three operation hours the lengthening rate stabilizes as is typical of the phase II of creep, (in this case $\sim 23 \mathrm{~mm} / \mathrm{h}$ ). This rate is observed also after shortening (red line Fig. 6) of the sublimators. For the new TaW2.5\% sublimators, the observed lengthening rate is around $1.2 \mathrm{~mm} / \mathrm{h}$, almost a factor of 20 less than for the standard sublimators, where no different creep phases have been witnessed yet.

The maximum operation time of 21 hours has been reached with the first pair of wires and so far over seven hours for the second pair. The progress of the wire elongation of the two new TaW2.5\% wires shows large oscillations. This is caused by a different power input in every pulse. The current control of the power supply has an accuracy of about $4 \%$ with strong

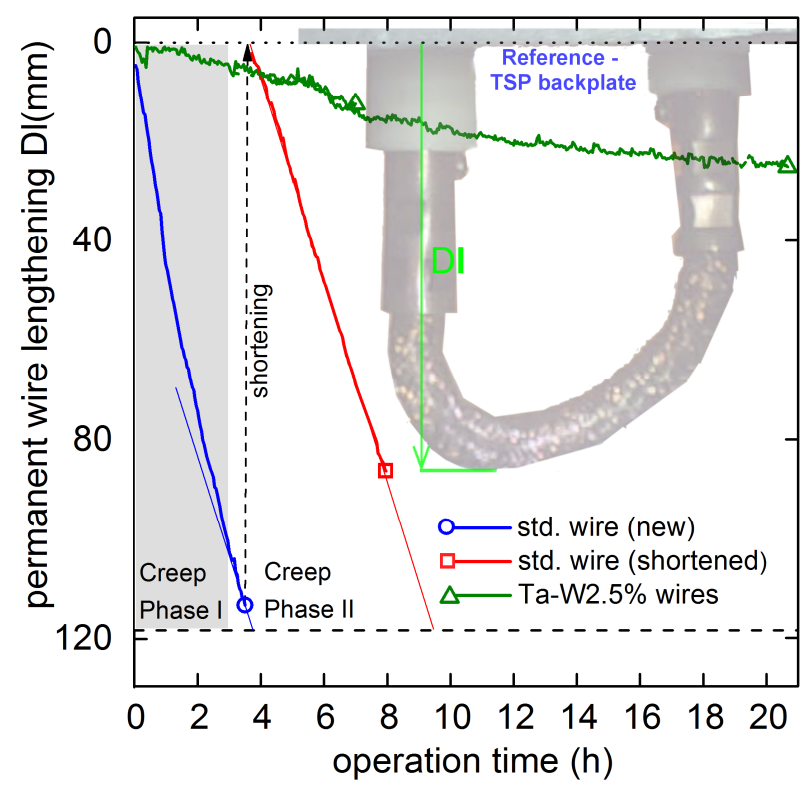

Fig. 6. Residual wire lengthening caused by material creep as a function of the operation time for new and shortened standard wires and the of the new $\mathrm{Ta}-\mathrm{W}$ core wires.

dependency on the operation frequency. In the future a power control of the power supply will be tried out so as to ensure a constant temperature that provides adequate $\mathrm{Ti}$ sublimation rates.

\section{B. Ti sublimators short-circuit mitigation}

The contact among sublimators reduces the TSP availability in terms of number of sublimators and TSP operation time, but also direct loss of pumping surface. One of the reasons of wire fatal contact may be the abrupt change of the current through the wires. As was visually observed in the test bed, suddenly switching the full current on or off induces strong wire oscillation due to the sudden shock caused by the abrupt onset of the electromagnetic force between the antiparallel currents in the wire pair. On the AUG-NBI TSPs the current is slowly ramped up and down at the beginning and end of the sublimation cycle to avoid this problem. However, the AUG main experiment control sometimes abruptly blocks TSP operation due to a magnetic field safety interlock that is meant to prevent simultaneous operation of the TSPs with the magnetic field coils of AUG. In this case the TSP current is abruptly set to zero. However, there is an inherent time delay between the interlock signal and the soonest possible start of AUG's magnetic fields. Hence there is enough time for a controlled ramp down of the TSP current. This has recently been implemented on AUG-NBI's TSPs. The effect will be observable during the next campaign.

During the tests for AC operation in a B-field there have been at least three cases of sublimator contact for the 12 tested pairs of wires. Special attention has been paid to identifying the causes. The accelerometer sensors have originally served to discard complications caused by resonant oscillations at certain frequencies during $\mathrm{AC}$ operation. A closer look at the 


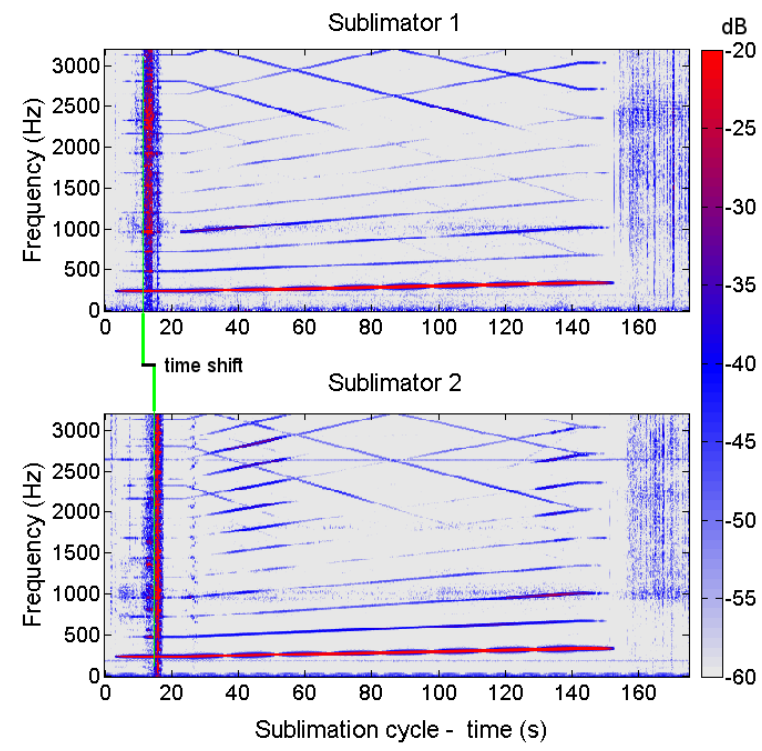

Fig. 7. STFT spectrogram of the acceleration sensors at the two sublimators of a pair during a regular heating cycle with a ramped frequency between $200-320 \mathrm{~Hz}$. The time shift between the sublimators suggests that they elongate at different speed due to probably different sublimator resistance.

spectrograms reveals a prolonged phase of noise (all frequency components, Fig. 7) in all four channels at the beginning and end of the heating cycle. The signal amplitude and duration are variable from cycle to cycle but coincide within the sensors attached to the same sublimators. The most straightforward explanation for this noise is the sliding contact during heating up and down of the insulating sleeves inside the guiding plate. If the friction is too large, it can be imagined that the heated sublimators elongate but the insulating sleeves do not slide through the guiding plate apertures. Thus the wires will bend sidewise and may come into contact. Three main causes have been identified that may lead to too high friction:

1. High friction coefficient for the sliding pair due to the choice of the materials, aluminum for the guiding plate and aluminum oxide for the insulation sleeve.

2. Excessively large contact forces may appear. The gap between the drillings of the guiding plate $(\varnothing 26 \mathrm{~mm})$ and the sliding ceramic insulator $(\varnothing 24 \mathrm{~mm})$ is quite big, while the thickness of the guiding plate is rather small $(30 \mathrm{~mm})$. This geometrical combination allows for an excessive tilting of the ceramic cylinder in the guide drilling and thus a large contact force in the guiding plate.

3. Different wire lengthening of each sublimator in a pair. This causes the $\mathrm{Cu}$ braid loop at the bottom to imprint a lateral force on the sublimators and the insulator sleeves may be tilted and pressed against the guiding plate.

In the new TSP design for W7-X, the sliding pair will be a material combination of bronze or stainless steel for the guiding plate and aluminum oxide for the sleeve. Furthermore, the gap between the insulator and the guiding plate will be reduced substantially. As the permanent lengthening of the new TaW2.5\% sublimators is less also the difference in wire lengthening with in a pair of sublimators will be accordingly less, leading to less tilting forces. A further improvement should be achieved by modifying the arrangement of the sublimators in the TSP. The distance between the three pairs of wires within one chamber has been increased by almost $30 \%$ in order to reduce fatal wire contacts.

\section{CONCLUSIONS AND OUTLOOK}

The design of new TSPs for the NBI injectors on W7-X has led to a series of improvements of the system, in preparation of the more demanding operation conditions for future long-pulse operation in W7-X (more than twice the TSP operation time on AUG). The availability of TSPs can be improved through reducing intrinsic lengthening of the Ti sublimators with a new supporting core made of a Ta alloy with $2.5 \% \mathrm{~W}$ in weight. Two pairs of these sublimators were tested so far with positive result. The electrical properties of the sublimators seem to be equivalent to the standard sublimators with unalloyed Ta. They are thus compatible with the available power supply and operation schema, which was a major constraint. The intrinsic lengthening is strongly reduced, allowing for enough operation time to completely deplete their Ti content without the need for intermediate sublimator shortening. A series of mechanical modifications were identified and will be implemented to reduce the hazard of sublimator short-circuits and consequent loss of pumping chamber. The need for maintenance is reduced considerably, allowing for extended operation time of the TSP of about a factor of 2.5. It should be sufficient for similarly long campaigns at W7-X as on AUG.

Tests of the new kind of sublimators are still in progress in order to gain more experience, while in a further next step, the focus will be set on optimizing the performance of the TSP, aiming at the better use of the $\mathrm{Ti}$ reservoir. To this goal a strategy must be developed first to study the pumping efficiency and capacity through the use of a calibrated gas feed and the existing pressure gauges. In a second stage, the influence of the Ti heating cycle's main parameters heating power and cycle duration will be studied in order to define a proper TSP operation scheme.

\section{ACKNOWLEDGMENTS} IPP.

Special thanks for the support to the ITED group staff at

\section{REFERENCES}

[1] G. Orozco, M. Froeschle, B. Heinemann, C. Hopf , R. Nocentini, R Riedl, A. Staebler,“ AC operation of large titanium sublimation pumps in a magnetic field: results of the test stand for the W7-X neutral beam injectors“, Fusion Eng. Des., Vol. 89, pp 3070-3077, September 2014.

[2] P. McNeely et al, "Current status of the neutral beam heating system of W7-X”, Fusion Eng. Des., Vol. 88, Issues 6-8, pp 1034-1037, March 2013.

[3] A. Staebler et al, "Design of the neutral beam injection system for ASDEX-Upgrade", Fusion Technology, pp. 620-624, 1988.

[4] H.-S. Bosch et al, "Technical challenges in the construction of the steady-state stellarator Wendelstein 7-X", Nucl. Fusion, Vol. 53, Issue 12, pp 12600, 2013. 
[5] J.-H. Feist, A. Staebler, W. Ertl, B. Heinemann, E. Speth, "Large scale titanium getter pumps for the ASDEX-Upgrade neutral beam injectors", Proc. 17th SOFT Fusion Technology, Vol. 1, pp 262-266, 1992.

[6] F. Prevot and Z. Sledziewski, "The titanium evaporation pump, its application to nuclear fusion experiments and space simulation", Vac. Sci. Technol., Vol. 9, pp 49, 1972.

[7] R.L. Hammel, R.E. Uhrig, "Creep of tantalum under cyclic elevated temperatures“, Ames Laboratory Technical Reports. Paper 14, 1960
[8] R. Titran, "Creep of tantalum T-222 alloy in ultrahigh vacuum for times up to 10000 hours", NASA technical note TN D-4605, May 1968.

[9] F.F. Schmidt, H.R. Ogden, "The engineering properties of tantalum and tantalum alloys", Defense metals information center, Battelle memorial institute, Report 189, 1963.

[10] P.D. Desai, T.K. Chu, H.M. James, C.Y. Ho, "Electrical resistivity of selected elements" J. Phys. Chem. Ref. Data 13, 1069, 1984 